diarrhea (86\%), anorexia (81\%), and vomiting (77\%). Mortality proportions among cases untreated and treated with vitamin A were $71.9 \%$ and $55.0 \%$, respectively. In a propensity-matched analysis, mortality was significantly lower among cases receiving vitamin A ( $R R=0.77$ 95\%; CI:0.59-0.99; $\mathrm{p}=0.041)$.

Discussion: Early vitamin A supplementation was associated with reduced mortality in EVD patients and should be provided routinely during future epidemics.

Prehosp. Disaster Med. 2019;34(Suppl. 1):s15-s16

doi:10.1017/S1049023X19000499

\section{Evolving Strategy and Incident Management Systems in Hard to Reach Areas and Fragile Security Settings: The Case of Ebola Response in the Democratic Republic of Congo Dr. Nda Konan Michel Yao ${ }^{1}$, Dr. Tambwe Bathe Ndjoloko ${ }^{2}$ 1. WHO AFRO, Brazzaville, Congo \\ 2. Ministry of Health, Kinshasa, Democratic Republic of Congo}

Introduction: DRC Ministry of Health declared the 9th outbreak of Ebola Virus Disease (EVD) in the Equator province on May 8, 2018, that ended on July 25, 2018. There were 54 cases with 38 confirmed, 33 deaths (61\%), and 21 survivors in three "zones de santé" (districts). On August 1, 2018, the 10th EVD outbreak of the country was declared in the Ituri and North Kivu provinces. This one is the most important outbreak ever experienced. By November 18, 2018, 373 cases were reported with 326 confirmed and 214 deaths (58\%) in two provinces including 14 "zones de santé." While the 9th outbreak occurred in hard-to-reach areas, the 10th is occurring in fragile security settings, requiring specific strategic/operational approaches.

Aim: To describe strategic and operational approaches including IMSs used to address these deadly outbreaks.

Methods: A case study methodology using response strategy documents and observations was used, coupled with the use of operation review exercises.

Results: The response strategy evolved continuously taking into account the epidemiological context, including geographical spread. It also took into account cultural, political, and sociological (community resistances) sensitivities. Conditions of pre-existing health system and services were considered. The prevailing security context (armed groups) was taken into account. The evolving situation impacted implementation of response areas including critical interventions like setting up confirmation and treatment centres, rapid response teams, and IMS structures. Areas of response were reviewed continuously, including response structures with further decentralization, outreach, or locally delegated interventions to ensure geographical access and continuity in response services.

Discussion: Response areas to deal with EVD outbreaks are well known. However, an effective response requires a continuous adjustment of the strategy and a flexible response structures with related IMSs based on regular deep situation analysis. Social sciences still have a critical role to play for that purpose.

Prehosp. Disaster Med. 2019;34(Suppl. 1):s16

doi:10.1017/S1049023X19000505 